\title{
Spectrometric mixture analysis: An unexpected wrinkle ${ }^{\dagger}$
}

\author{
ROBERT DE LEVIE \\ Chemistry Department, Bowdoin College, Brunswick ME 04011, USA \\ e-mail: rdelevie $@$ bowdoin.edu
}

\begin{abstract}
The spectrometric analysis of a mixture of two chemically and spectroscopically similar compounds is illustrated for the simultaneous spectrometric determination of caffeine and theobromine, the primary stimulants in coffee and tea, based on their ultraviolet absorbances. Their analysis indicates that such measurements may need an unexpectedly high precision to yield accurate answers, because of an artifact of inverse cancellation, in which a small noise or drift signal is misinterpreted in terms of a concentration difference. The computed sum of the concentrations is not affected.
\end{abstract}

Keywords. Spectrometric mixture analysis; caffeine; theobromine; ultraviolet absorption.

\section{Introduction}

The analysis of mixtures is often used in undergraduate textbooks and laboratory exercises to illustrate how to handle the complexity of actual analytical samples. While, in practice, one can often avoid mixture analysis by using efficient separation methods such as provided by chromatography, separations may not always be possible or convenient, as in automated process control. Spectroscopic measurements are typically non-destructive, and can often be made 'on the fly' rather than requiring that discrete samples be taken. In many cases, spectroscopic mixture analysis without prior separation may therefore be desirable. In kinetic measurements, e.g. it is often much easier to analyse reagents, intermediates, and/or products continuously by spectroscopy rather than to sample the reaction mixture, stop the reaction from progressing, separate the mixture into its individual components, and then determine their concentrations separately. In the present communication, we will consider an analysis based on external calibration measurements. Started as an effort to see how far one can push this method, we come up with what (at least to us) is an unexpected wrinkle, which limits what one can achieve.

In principle, there are two ways to perform a mixture analysis: one can either use a minimally determined or an overdetermined system. In a minimally determined system one makes exactly as many (or, rather, as few) measurements as there are unknowns.

${ }^{\dagger}$ Dedicated to the memory of the late Professor S K Rangarajan
For the spectrometric determination of two components in a binary mixture, one then measures, e.g. the absorbance (or a quantity directly proportional to it, such as its first or higher derivative) at two different wavelengths; for a ternary mixture one records an optical measure proportional to the sought concentrations at three different wavelengths, etc. The resulting calculation is simple, and typically yields a determinant ratio which, at least for a small number of mixture components, can be evaluated readily with Cramer's rule.

When the measurements contain a significant amount of random noise, one might prefer another approach, just as, in that case, it would be better to use a multi-point calibration graph rather than a single calibration point for determining the concentration of a single unknown concentration. When one uses only a minimal number of measurements, the input data are implied to be error-free, because any experimental errors will be transferred fully into the final results. Moreover, the response must follow a strict proportionality, because that is implicitly assumed as well. When more than the absolute minimum of data points are taken, the effect of noise can be reduced, internal consistency checks can be made, and one can verify whether the assumed model (in this case, Beer's law) is applicable and, in case it is not, adjust the analysis model accordingly. (This latter aspect will not be illustrated here because, in the present example, we found no significant deviations from Beer's law. But this was a conclusion based on experimental evidence, rather than an unverified assumption.) 
In practice it would therefore seem preferable to use an overdetermined system, in which one collects many more data than strictly needed. Overdetermined systems typically require the use of some computer program, such as a least squares routine, but these are now so ubiquitous that this can no longer be considered much of a constraint.

In the present communication, we will compare the use of a minimally determined and an overdetermined analysis approach, based on the nearultraviolet absorption of two common chemicals, caffeine and theobromine. Caffeine is the major stimulant in coffee and tea, as well as in many other beverages, while theobromine is the main stimulant in chocolate, and is also present in tea. Somewhat perversely, caffeine is now readily available to softdrink manufacturers as a by-product of decaffeinating coffee.

Caffeine (1,3,7-trimethylxanthine) and theobromine (3,7-dimethylxanthine) only differ by one methyl group. As can be seen from figure 1, their ultraviolet spectra are quite similar, with peaks at virtually the same wavelengths, differing only slightly in their molar absorptivities. This makes their simultaneous spectroscopic determination nontrivial.

Caffeine and theobromine are chemically fairly stable, although their aqueous solutions can be airoxidized slowly. We have therefore worked with

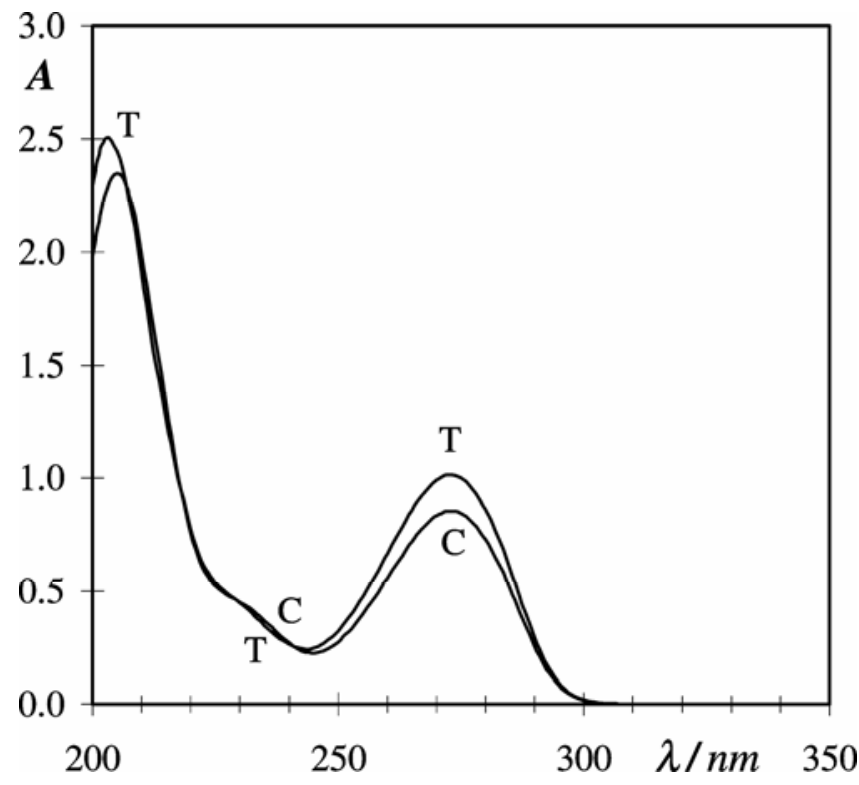

Figure 1. The absorbance of $100 \mu \mathrm{M}$ caffeine (curve C) and $100 \mu \mathrm{M}$ theobromine (curve $\mathrm{T}$ ) in aqueous $0.05 \mathrm{M}$ $\mathrm{KH}_{2} \mathrm{PO}_{4}+0.05 \mathrm{M} \mathrm{Na}_{2} \mathrm{HPO}_{4}$. freshly made solutions, stored under nitrogen or argon. Their molar absorptivities in the ultraviolet region of the spectrum are quite considerable (of the order of $10^{4} \mathrm{M}^{-1} \mathrm{~cm}^{-1}$ at $273 \mathrm{~nm}$, see figure 1), so that in ultraviolet absorption spectrometry they must typically (i.e. in cells with an optical pathlength of $1 \mathrm{~cm}$ ) be used in quite dilute solutions, well below $1 \mathrm{mM}$, at which level they are quite innocuous. (The concentration of caffeine in our stock solution is only about 1.5 times that in Coca Cola, while those in the solutions used for the actual spectrometry are at least five times lower, i.e. they are always less than $30 \%$ of that of Coca Cola.) Because the spectra of the two light-absorbing components are so similar, these mixtures should provide a good test of the factors that define spectroscopic precision.

\section{Beer's law for mixtures}

When the absorption of a single species follows Beer's law, we can write

$$
A=a b c,
$$

where $A$ is the measured absorbance, $a$ the molar absorptivity (or extinction coefficient), $b$ the optical path length, and $c$ the concentration of the absorbing species.

For a solution containing two chemically distinct, non-interacting, light-absorbing species, here labelled 1 and 2, we then have

$$
A=a_{1} b c_{1}+a_{2} b c_{2}
$$

where $b$ does not carry an index because the lightabsorbing species in a mixture share the same cell, and therefore the same optical pathlength $b$. For a multicomponent mixture of $i$ light-absorbing constituents we have, likewise,

$$
A=\sum_{i} a_{i} b c_{i}
$$

When we have access to standards for each of the sample components, we can measure their individual absorbance spectra as

$$
A_{i}=a_{i} b C_{i},
$$

where $C_{i}$ denotes the concentration of the individual standard used for component $i$. By introducing the 
concentration quotients $q_{i}=c_{i} / C_{i}$ we can then combine (3) and (4) to

$$
A=\sum_{i} a_{i} b q_{i} C_{i}=\sum_{i} q_{i} A_{i},
$$

which expresses the absorbance $A$ of the mixture in terms of the absorbances of the individual standards $A_{i}$ and the concentration ratios $q_{i}=c_{i} / C_{i}$. It is in this form that we will analyse our data. Note that the absorbance $A$ of the mixture, and the individual absorbances $A_{i}$ of the standards, are functions of wavelength or wavenumber, whereas the concentration quotients $q_{i}$ are single-valued numbers.

When least squares are used to fit the proportionality $y=q x$ to a set of data $y(x)$, one has two functions, $y$ and $x$, and a single adjustable parameter, the slope $q$. In the corresponding multivariate case, $y$ will depend on several functions $x_{i}$, and one therefore uses

$$
y=\sum_{i} q_{i} x_{i} .
$$

In such a least squares analysis, the functions $y$ and $x_{i}$ are simply sets of numbers, rather than explicit mathematical expressions. Numerical analysis, including that by least squares, actually makes no such distinction, and treats all functions merely as sets of numbers. Given the formal correspondence between (5) and (6), one can therefore use a standard multivariate least squares routine to fit the model expression (5) to a mixture absorbance $A$, using the standard spectra $A_{i}$ as the 'independent' or 'control' variables. This will directly yield the corresponding concentration ratios $q_{i}$, as well as the corresponding uncertainty estimates. By using this overdetermined approach, random noise should be reduced by a factor of $(N / P)^{1 / 2}$, where $N$ is the number of independent spectral measurements, and $P$ the number of extracted parameters.

For our binary mixture (i.e. $P=2$ ) we have used absorbance measurements at $1 \mathrm{~nm}$ intervals over a $80 \mathrm{~nm}$ range, so that (assuming that these are independent measurements) we can anticipate at most about a nine-fold reduction in random noise. In fact, the slit width was set at $2 \mathrm{~nm}$, so that the number of independent data points was only 40 , and the resulting maximal noise reduction only a factor of about six. Of course, systematic errors are not reduced, i.e. this argument only concerns precision, not accuracy.
Alternatively, one can select a number of wavelengths equal to the number of absorbing mixture components, and solve the resulting simultaneous equations. For a mixture of two absorbing species, measured at wavelengths ' and " respectively, we then have

$$
\begin{aligned}
& A^{\prime}=a_{1}^{\prime} b c_{1}+a_{2}^{\prime} b c_{2}=q_{1} A_{1}^{\prime}+q_{2} A_{2}^{\prime}, \\
& A^{\prime \prime}=a_{1}^{\prime \prime} b c_{1}+a_{2}^{\prime \prime} b c_{2}=q_{1} A_{1}^{\prime \prime}+q_{2} A_{2}^{\prime \prime}
\end{aligned}
$$

from which we obtain

$$
\begin{aligned}
& q_{1}=\frac{\left|\begin{array}{ll}
A^{\prime} & A_{2}^{\prime} \\
A^{\prime \prime} & A_{2}^{\prime \prime}
\end{array}\right|}{\left|\begin{array}{ll}
A_{1}^{\prime} & A_{2}^{\prime} \\
A_{1}^{\prime \prime} & A_{2}^{\prime \prime}
\end{array}\right|}=\frac{A^{\prime} A_{2}^{\prime \prime}-A_{2}^{\prime} A^{\prime \prime}}{A_{1}^{\prime} A_{2}^{\prime \prime}-A_{2}^{\prime} A_{1}^{\prime \prime}}, \\
& q_{2}=\frac{\left|\begin{array}{cc}
A_{1}^{\prime} & A^{\prime} \\
A_{1}^{\prime \prime} & A^{\prime \prime}
\end{array}\right|}{\left|\begin{array}{ll}
A_{1}^{\prime} & A_{2}^{\prime} \\
A_{1}^{\prime \prime} & A_{2}^{\prime \prime}
\end{array}\right|}=\frac{A_{1}^{\prime} A^{\prime \prime}-A^{\prime} A_{1}^{\prime \prime}}{A_{1}^{\prime} A_{2}^{\prime \prime}-A_{2}^{\prime} A_{1}^{\prime \prime}} .
\end{aligned}
$$

In what follows we will consider both the overdetermined, least squares based method, and the minimally determined method based on the determinant ratios, (8).

\section{Precautions}

The major known error sources in a spectrophotometric project like this are (i) volumetric errors in preparing the solutions, (ii) irreproducibility of cuvet placement, and (iii) gradual changes in environmental and/or instrumental parameters, such as the ambient temperature and (possibly related) instrumental baseline drift. In order to reduce such errors, we have used a motor-driven precision pipet instead of a manual one, and a stationary flow-through cell, and have made measurements on a precision instrument in an airconditioned lab. These precautions indeed reduced the corresponding errors. Using a flow-through cell introduces the possibility of sample carry-over from one experiment to the next as the result of insufficient solution flushing, and we were careful to minimize that error. The measurements shown here were all obtained in a single, oneday measurement session. 


\section{Chemicals}

Caffeine was obtained from Aldrich, and theobromine from Sigma. They were used as such, without further purification. As methyl-substituted xanthines, both caffeine and theobromine have acid-base equilibria that make their ultraviolet spectra potentially $\mathrm{pH}$-dependent. For a quantitative application of Beer's law we must therefore maintain a constant $\mathrm{pH}$. Consequently, we have made all our solutions and measurements in a neutral aqueous phosphate buffer composed of $0.05 \mathrm{M} \mathrm{KH}_{2} \mathrm{PO}_{4}+0.05 \mathrm{M} \mathrm{Na}_{2} \mathrm{HPO}_{4}$.

\section{Volumetrics}

Three stock solutions were made, one of the $0.05 \mathrm{M}$ $\mathrm{KH}_{2} \mathrm{PO}_{4}+0.05 \mathrm{M} \mathrm{Na}_{2} \mathrm{HPO}_{4}$ phosphate buffer, one of $1.00 \mathrm{mM}$ caffeine in that phosphate buffer, and one of $1.00 \mathrm{mM}$ theobromine in the same phosphate buffer. Starting from these three stock solutions, we then made a number of standard solutions by volumetric mixing of these stock solutions in order to make $100 \mathrm{~mL}$ volumes of standards that contained only caffeine or theobromine, and a number of synthetic samples containing caffeine, theobromine, or both, all in the same phosphate buffer. The distinction between standards and one-component samples is purely by assignment.

In order to minimize pipetting errors, we made all our dilutions by using a Metrohm Dosimat model 775 liquid dispenser, a motor-driven precision pipet outfitted with a $1 \mathrm{~mL}$ buret, which we used to deliver an integer number of milliliters (i.e. buretfuls) of stock solution into $100 \mathrm{~mL}$ volumetric flasks. This gave us a reproducibility of the delivered volumes of at least $\pm 0.001 \mathrm{~mL}$, i.e. to at least $\pm 0.1 \%$, approximately three times better than we could achieve with a regular, manual pipet.

The $100 \mathrm{~mL}$ volumetric flasks were then filled manually to the mark with the buffer stock solution. We estimate the resulting uncertainty to be of the order of two drops, i.e. $\pm 0 \cdot 1 \mathrm{~mL}$, again corresponding to about $\pm 0 \cdot 1 \%$. The volumetric flasks used were either A and B grade, and therefore had a volumetric accuracy of about $0 \cdot 15 \%$. A conservative estimate of the over-all volumetric imprecision is therefore of the order of $\pm 0 \cdot 2 \%$.

As will be indicated below, there was most probably another possible error source, namely the occasional, unintended transfer of some stock solution from the outside of the delivery tip of the dis- penser into one or more standards and/or samples, equivalent to a partial drop carried on the outside of a pipet. Between preparing individual standard and sample solutions, we touched the delivery tip to the walls of a clean receptor vessel in order to remove any adhering solution, and during delivery we made sure that the delivery tip made direct contact with the inside wall of the receiving vessel, so that partial drops would be delivered properly, but these precautions may have failed on occasion. However, since a possibly resulting error was only identified after the fact, i.e. during the data analysis, we did not exclude any such suspect data from the analysis.

We made two sets of standard solutions, containing $0,2,4,6,8,10,12,14,16$, and $18 \mathrm{~mL}$ of either caffeine or theobromine stock solution respectively per $100 \mathrm{~mL}$ of sample. These therefore correspond to $0,20,40,60,80,100,120,140,160$, and $180 \mu \mathrm{M}$ concentrations. We made 36 samples, containing 0 , $1,3,5,7$, or $9 \mathrm{~mL}$ of caffeine and/or theobromine. We will refer to these solutions in terms of the resulting micromolar concentrations, i.e. ten times the number of milliliters of stock solutions used in preparing each of them, with $c$ denoting that micromolar concentration for caffeine, and $t$ that of theobromine, so that, e.g. a sample with $c=50$ and $t=70$ identifies a solution prepared with $5 \mathrm{~mL}$ caffeine stock solution and $7 \mathrm{~mL}$ theobromine stock solution, made up to $100 \mathrm{~mL}$ with buffer, i.e. $50 \mu \mathrm{M}$ in caffeine and $70 \mu \mathrm{m}$ theobromine. Note that all of these standard and sample solutions are made from the same three stock solutions: caffeine in buffer, theobromine in buffer, and buffer alone.

\section{Spectrometry}

The absorbance of each solution was measured with a Varian Cary 400 double-beam spectrometer between 200 and $350 \mathrm{~nm}$, at $1 \mathrm{~nm}$ intervals and a sampling rate of one absorbance measurement per second. The absorbances were stored, and subsequently imported into an Excel spreadsheet. The usual precautions were taken, such as turning the instrument on several hours before the start of measurements in order to let it reach temperature equilibrium. There was no switching of lamps or gratings in the wavelength range used in our analysis.

The solution volume in the $(1 \times 1 \times 5 \mathrm{~cm}$, standard size) cell and its associated Teflon spaghetti tubing was about $3 \mathrm{~mL}$, and was completely flushed out with $30 \mathrm{~mL}$ of a fresh solution. As an additional 
precaution we interspersed all measurements by flushing the cell with $30 \mathrm{~mL}$ aliquots of buffer, so that the actual protocol used a $30 \mathrm{~mL}$ rinse with buffer, then a $30 \mathrm{~mL}$ rinse with the sample, followed by the spectroscopic measurement. Flushing could be done with a simple syringe outfitted with a threeway stopcock or, more conveniently, with a peristaltic pump, to which connections were made with flexible, narrow-bore Teflon tubing. The pump was placed between the cell and a waste container, and its tygon tubing came only in contact with the solution after it had been measured, on its way to be discarded. For the measurements shown, we used an Ismatec Reglo Digital peristaltic pump which was set to deliver $30 \mathrm{~mL}$ aliquots, in about $150 \mathrm{~s}$. After every five or six measurements the baseline was rerun and the zero levels reset accordingly.

While our motivation in using the flow-through cell was to reduce cell positioning errors, we found that it is actually simpler for making an extended set of serial measurements, and certainly not slower, than removing, rinsing, refilling, and reinserting cuvets. However, it required that we improvise a simple, light-tight cover for the cell compartment in order to allow the two connecting tubes to enter that compartment without introducing stray light. All solutions were measured in a single, fixed-position flow-through cell with a $1 \mathrm{~cm}$ optical pathlength.

During the experiment, the lab temperature varied from 21.6 to $23 \cdot 0^{\circ} \mathrm{C}$, and baseline stability was well within \pm 0.01 absorbance units, except at wavelengths below $220 \mathrm{~nm}$, which were therefore excluded from the analysis.

At $350 \mathrm{~nm}$ the absorbance recorded as zero for all our solutions, and this was therefore used to zero the instrument just before each individual spectrum was run. The instrument measures the absorbance to a claimed resolution of 0.001 absorbance units; its photometric accuracy is given by the manufacturer as \pm 0.004 absorbance units at $A=1$, its photometric repeatability as \pm 0.002 , its baseline stability and flatness as both $\pm 0 \cdot 001$. We can therefore expect our measurements to be reproducible to within a few thousands of an absorbance unit, and control measurements indeed bear this out.

In measuring the standard solutions, we interspersed and measured blank solutions after four or five measurements, and readjusted the baseline accordingly, and we did the same between various sets of sample measurements. For details, see the Supporting Information, which contains the actual measurements and their complete analysis.

\section{Preliminary data analysis}

In order to test the applicability of Beer's law, we first use the two sets of 10 standard solutions, containing $0,2,4,6,8,10,12,14,16$, and $18 \mathrm{~mL}$ of either caffeine or theobromine stock solution respectively per $100 \mathrm{~mL}$ of sample, corresponding to $0,20,40,60$, $80,100,120,140,160$, and $180 \mu \mathrm{M}$ concentrations.

Upon dividing the absorption spectra obtained for these standard solutions by $10^{6}$ times their $c$ - or $t$ values, the curves for all 8 solutions containing only theobromine, i.e. $(0,20),(0,40),(0,80),(0,100)$, $(0,120),(0,140),(0,160)$, and $(0,180)$, essentially coincide to within the linewidths of the curves, see figure A-7 in the Supporting Information, and the same applies to the caffeine data except for one curve, $(20,0)$, which is about $1 \%$ higher than the others, see figure A-8. The latter is equivalent to a possible dosage error of $0.02 \mathrm{~mL}$, such as might have been made when a partial droplet of caffeine stock solution is carried on the delivery tip of the automatic pipet. At higher concentrations, such an experimental error would be less consequential. Note that the vertical scales in figures A-7 and A-8 are the molar absorptivity $a$, in units of $\mathrm{M}^{-1} \mathrm{~cm}^{-1}$. Such a good (visual) proportionality of the various curves suggests that Beer's law indeed applies.

For a more quantitative check, we fit the model expressions $A=a_{1} c$ and $A^{\prime}=a_{0}+a_{1} c+a_{2} c^{2}$ to the absorbances at $273 \mathrm{~nm}$ of all caffeine standard solutions and, separately, for all standard theobromine solutions, in both cases with $c$ in $\mu \mathrm{M}$. For caffeine, with the model expression $A=a_{1} c$ we find $a_{1} \pm s_{1}=$ $0.0085490_{4} \pm 0.0000005_{7}$, where the second number indicates the corresponding standard deviation, $s_{1}$, while the standard deviation of the overall fit, $s_{f}$ is $0.0001_{9}$. With the expression $A^{\prime}=a_{0}+a_{1} c+a_{2} c^{2}$ we obtain $a_{0} \pm s_{0}=-0.0001_{3} \pm 0.0002_{6}, \quad a_{1} \pm s_{1}=$ $0.008549_{5} \pm 0.000005_{9}, \quad$ and $a_{2} \pm s_{2}=4 \cdot_{1} \times 10^{-9} \pm$ $2 \cdot{ }_{9} \times 10^{-8}$, with $s_{f}=0.0002_{0}$. Because $s_{0}$ is larger than $\left|a_{0}\right|$, the offset term $a_{0}$ is statistically insignificant, and the same applies to the quadratic term $a_{2}$. Consequently, Beer's law is not only the theoretically predicted model, but is also the experimentally found one. There are, of course, an infinite number of possible models, but a non-zero offset and a quadratic term are usually the first-order indications of deviations from a strict proportionality, and they are clearly absent here.

For theobromine, the model expression $A=a_{1} c$ yields $a_{1} \pm s_{1}=0.010146_{0} \pm 0.000005_{9}, s_{f}=0.002_{0}$. 
With $A^{\prime}=a_{0}+a_{1} c+a_{2} c^{2}$ we find $a_{0} \pm s_{0}=0.002_{4} \pm$ $0.002_{0}, \quad a_{1} \pm s_{1}=0.01013_{8} \pm 0.00004_{5}, \quad a_{2} \pm s_{2}=$ $-8.0 \times 10^{-8} \pm 2.2 \times 10^{-7}$, and $s_{f}=0.001_{5}$. Again, $s_{0}$ is almost as large as $\left|a_{0}\right|$, and $s_{2}$ is larger than $\left|a_{2}\right|$, indicating as before that these coefficients are not statistically significant. (A reasonable criterion of statistical significance would be that $s_{i} /\left|a_{0}\right|$ be larger than 3; a conservative one might require that the ratio $s_{i} /\left|a_{0}\right|$ exceeds 5.) We therefore conclude that Beer's law holds for both compounds under the experimental conditions and for the concentration range investigated here.

For a quick visual check of the mixture spectra, we plotted the absorbances of those solutions that have a constant value of $c+t$, such as $(0,180),(90$, $90)$, and $(180,0)$, where $c+t=180$. Likewise we plotted the absorbances for $c+t=160$, i.e. curves 0 , $160,70,90,90,70$, and 160, 0 ; at $c+t=140$ with curves $(0,140),(50,90),(70,70),(90,50)$, and $(140,0)$; at $c+t=120$ for the mixtures $(0,120)$, $(30,90),(50,70),(70,50),(90,30)$, and $(120,0)$; at $c+t=100$ for curves $(0,100),(1,90),(3,70),(50$, $50),(70,30),(90,10)$, and $(100,0)$; and likewise for $c+t=80$ (6 different curves), 60 (5 curves), 40 (4 curves), and 20 ( 3 curves). All of these are displayed in figure 2, which focuses on the region from 220 to $250 \mathrm{~nm}$, and shows that all these curves go through isosbestic points at 229 and $241 \mathrm{~nm}$, at which wavelengths the molar absorbances of caffeine and theobromine are therefore the same within the resolution of the display. Where they exist, isosbestic points are often useful as rough indicators of data quality. Note also that, in the region between 220 and $245 \mathrm{~nm}$, the spectral differences between mixtures at constant $c+t$ are rather small, adding to the challenge of this analysis.

Now that we know that the absorbance strictly follows Beer's law, i.e. a proportionality between absorbance $A$ and concentration $C$, we compute the average caffeine standard spectrum at each wavelength $\lambda$ by using the least squares formalism for such a proportionality, i.e. as

$$
\begin{aligned}
& A_{c, \text { standard }}(\lambda)=\frac{\sum c A_{c}(\lambda)}{\sum c^{2}}, \\
& A_{t, \text { standard }}(\lambda)=\frac{\sum t A_{t}(\lambda)}{\sum t^{2}},
\end{aligned}
$$

for the two sets of standard solutions. The two resulting standard curves will be our reference for caffeine and theobromine respectively at $1 \mu \mathrm{M}$ concentrations.

\section{Computing the mixture concentrations}

For the overdetermined method we use the remaining sample measurements, which comprise two groups: five solutions each with caffeine or theobromine only, and 25 with both components present, for a total of 35 samples, interspersed by 12 baseline measurements. We will first analyse all mixtures as if they contain both components.

The actual data analysis is quite straightforward. ${ }^{1}$ We make three columns, in the first of which we place the 81 data of a particular sample, as measured between 220 and $300 \mathrm{~nm}$, and in the second and third we permanently place the corresponding standard curves for caffeine and theobromine over the same wavelength interval. We then apply a least squares analysis, where the sample curve is the 'dependent' or 'response' variable, $y$, the two standard curves the 'independent', 'explanatory', or 'control' variables, $x_{1}$ and $x_{2}$; in this case, $c=x_{1}$ and $t=x_{2}$.

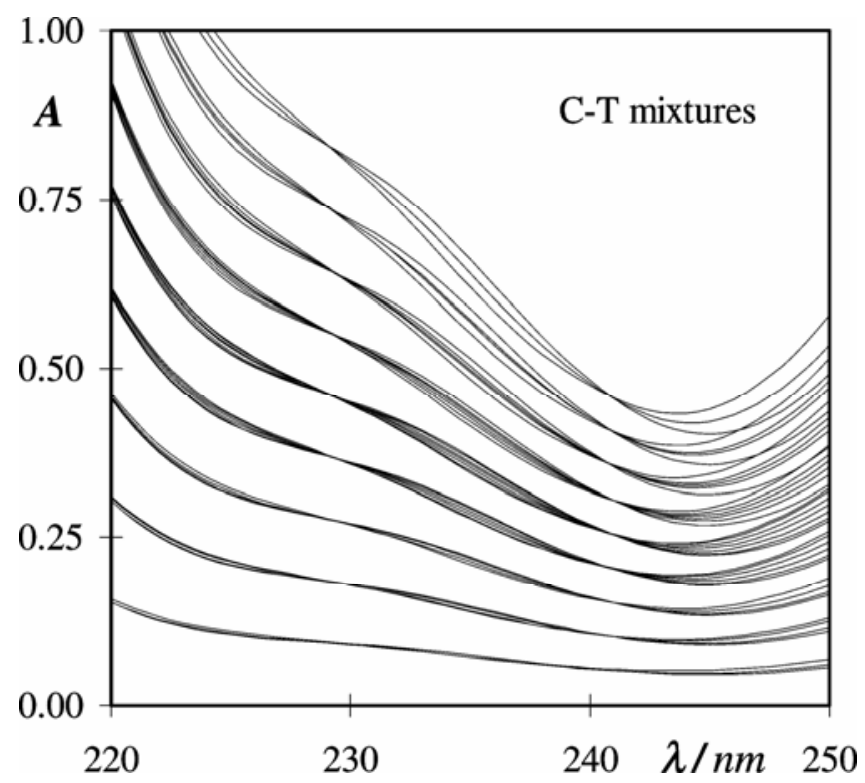

Figure 2. The absorbances of all mixtures of caffeine and theobromine in aqueous $0.05 \mathrm{M} \mathrm{KH}_{2} \mathrm{PO}_{4}+0.05 \mathrm{M}$ $\mathrm{Na}_{2} \mathrm{HPO}_{4}$ with (from top to bottom) $c+t=180,160,140$, $120,100,80,60,40$, and 20 , between 220 and $250 \mathrm{~nm}$. The differences of caffeine and theobromine absorbance between 250 and $300 \mathrm{~nm}$ are more pronounced, but exhibit no isosbestic points. 
We record the answers, which will be directly in micromolar concentrations, replace the sample curve by the next, and repeat the process. Since we have earlier established that Beer's law applies, the least squares analysis uses a strict proportionality, without intercept or terms of order higher than 1, i.e. the fitting model function is $y=a_{1} x_{1}+a_{2} x_{2}$, where $a_{1}$ and $a_{2}$ are micromolar concentrations. Of course, we can do this only because the standards and samples are all made up from the same stock solutions, and because we had earlier established that both standards indeed fit this model.

For the least squares analysis we use the Excel function LinEst, while a data line close to the analysis column extracts all the needed information from LinEst, and makes the associated computations. The resulting values are then copied with Edit $>$ Paste $\underline{\text { Special }}>$ Values into a final data table, from which tables 1-5 were subsequently extracted. For each sample we therefore only need to copy the data set into the analysis column, and copy the resulting data line with numerical values into the appropriate table. Everything else the spreadsheet does automatically for us, updating the data line every time we enter a new data set into the analysis column.

For the minimally determined analysis we use two wavelengths. The first of these is $273 \mathrm{~nm}$, where both caffeine and theobromine spectra show peak maxima, and where the molar absorptivity of theobromine is about $17 \%$ larger than that of caffeine. For the second wavelength we use $236 \mathrm{~nm}$, where the molar absorptivity of caffeine exceeds that of theobromine by about $6 \%$, see figure 2 . We use (8) to compute the mixture composition. Again, these results are computed in the data line and copied, at the same time as the least squares data, into the appropriate table.

Tables 1-3 list the main results of this analysis; the master table in the Supporting Information includes some additional information. Since these are carefully made-up samples, we show the absolute deviations from the nominal concentrations (i.e. from what we believe the concentrations to be) $\Delta c$ and $\Delta t$ as well as, in the case of the least squares analysis, the standard deviations $s_{c}$ and $s_{t}$. The latter are consistently much smaller, and are therefore rather suspect. We have already indicated that there will be errors in our assignment of the nominal concentrations, such as the apparent error of $+0.2 \mu \mathrm{M}$ in solution $(20,0)$, but these nominal concentrations are still the most reliable we have.
For the 12 baseline solutions shown in table 1 we find a standard deviation of the $\Delta c$ and $\Delta t$ terms obtained by least squares of the order of $0.2 \mu \mathrm{M}$, and slightly larger for the determinant method. For the five sample solutions containing only caffeine (see table 2) the corresponding numbers are about 0.16 and $0.13 \mu \mathrm{M}$; for only theobromine, they are about 0.31 and $0.15 \mu \mathrm{M}$ respectively, i.e. considerably worse for the least squares method. For the 25 samples that contain both caffeine and theobromine (see table 3) the standard deviations of the $\Delta c$ and $\Delta t$ terms range from 0.3 to $0.5 \mu \mathrm{M}$ for caffeine, and from 0.2 to $0.4 \mu \mathrm{M}$ for theobromine, with both methods. The standard deviation of all $130 \Delta c$ and $\Delta t$ measurements with the least squares approach is $0.32 \mu \mathrm{M}$, while the same measure for the determinant method yields $0.33 \mu \mathrm{M}$. Consequently, there does not appear to be much of a systematic difference between the two methods, perhaps reflecting the fact that the advantage of the least squares method lies in its greater immunity against random noise, which in these data does not seem to be the major source of errors (see below). There also does not appear to be a strong correlation between the magnitudes of the errors and the concentrations, although there is some trend towards larger errors with larger nominal concentrations.

Using three standard deviations as our guide, we conclude that the caffeine and theobromine concentrations in their mixtures between 0 and $100 \mathrm{mM}$, with standard deviations of about $0.33 \mu \mathrm{M}$, have a $99 \%$ confidence level of about $\pm 1 \mu \mathrm{M}$. These are some-what disappointing results, considering the

Table 1. The results for the analysis of all baseline samples $(0,0)$ in terms of caffeine and theobromine.

\begin{tabular}{|c|c|c|c|}
\hline \multicolumn{2}{|c|}{$\begin{array}{l}\text { Results from least } \\
\text { squares }\end{array}$} & \multicolumn{2}{|c|}{$\begin{array}{c}\text { Results from } \\
\text { determinant ratio }\end{array}$} \\
\hline c found & $t$ found & c found & $t$ found \\
\hline-0.0031 & 0.0042 & -0.0083 & 0.0098 \\
\hline $0 \cdot 0782$ & $-0 \cdot 0861$ & 0.0337 & -0.0335 \\
\hline 0.0606 & -0.0527 & -0.0314 & 0.0261 \\
\hline-0.0595 & $0 \cdot 0585$ & -0.1572 & $0 \cdot 1514$ \\
\hline $0 \cdot 1018$ & -0.0927 & $-0 \cdot 0021$ & 0.0027 \\
\hline-0.2574 & $0 \cdot 1942$ & $-0 \cdot 5518$ & 0.4413 \\
\hline 0.0161 & -0.0536 & -0.0584 & $0 \cdot 0126$ \\
\hline 0.1132 & -0.1479 & $0 \cdot 3446$ & -0.3451 \\
\hline-0.0733 & $0 \cdot 0606$ & $-0 \cdot 1627$ & $0 \cdot 1340$ \\
\hline-0.0198 & 0.0142 & $-0 \cdot 1776$ & $0 \cdot 1464$ \\
\hline $0 \cdot 1184$ & -0.0802 & $0 \cdot 1990$ & -0.1528 \\
\hline 0.6644 & -0.5972 & $0 \cdot 5161$ & -0.4850 \\
\hline
\end{tabular}


Table 2. Results for the analysis of the mixed sample solutions containing both theobromine and caffeine. $\Delta c=c$ found $-c$ nom, and $\Delta t=t$ found $-t n o m$, where $n o m$ refers to the nominal concentration.

\begin{tabular}{|c|c|c|c|c|c|c|c|c|c|}
\hline \multicolumn{5}{|c|}{ Results from least squares } & \multicolumn{5}{|c|}{ Results from determinant ratio } \\
\hline c found & $t$ found & $\Delta c$ & $\Delta t$ & c nom & t nom & c found & t found & $\Delta c$ & $\Delta t$ \\
\hline $9 \cdot 744$ & $10 \cdot 230$ & $-0 \cdot 256$ & $0 \cdot 230$ & 10 & 10 & $9 \cdot 849$ & $10 \cdot 111$ & $-0 \cdot 151$ & $0 \cdot 111$ \\
\hline $9 \cdot 566$ & $30 \cdot 422$ & $-0 \cdot 434$ & 0.422 & 10 & 30 & $9 \cdot 534$ & $30 \cdot 407$ & $-0 \cdot 466$ & $0 \cdot 407$ \\
\hline $10 \cdot 174$ & $49 \cdot 977$ & $0 \cdot 174$ & $-0 \cdot 023$ & 10 & 50 & $10 \cdot 319$ & $49 \cdot 775$ & $0 \cdot 319$ & $-0 \cdot 225$ \\
\hline $9 \cdot 390$ & $70 \cdot 786$ & $-0 \cdot 610$ & 0.786 & 10 & 70 & $9 \cdot 440$ & $70 \cdot 675$ & $-0 \cdot 560$ & 0.675 \\
\hline $10 \cdot 338$ & $89 \cdot 880$ & $0 \cdot 338$ & $-0 \cdot 120$ & 10 & 90 & $10 \cdot 515$ & $89 \cdot 660$ & $0 \cdot 515$ & $-0 \cdot 340$ \\
\hline $30 \cdot 039$ & 9.904 & 0.039 & -0.096 & 30 & 10 & $30 \cdot 218$ & $9 \cdot 704$ & $0 \cdot 218$ & $-0 \cdot 296$ \\
\hline 29.853 & $30 \cdot 094$ & $-0 \cdot 147$ & $0 \cdot 094$ & 30 & 30 & $29 \cdot 966$ & 29.936 & -0.034 & $-0 \cdot 064$ \\
\hline $30 \cdot 335$ & $49 \cdot 880$ & $0 \cdot 335$ & $-0 \cdot 120$ & 30 & 50 & $30 \cdot 441$ & $49 \cdot 720$ & $0 \cdot 441$ & $-0 \cdot 280$ \\
\hline $30 \cdot 397$ & 69.734 & $0 \cdot 397$ & $-0 \cdot 266$ & 30 & 70 & $30 \cdot 358$ & $69 \cdot 705$ & $0 \cdot 358$ & $-0 \cdot 295$ \\
\hline $29 \cdot 748$ & $90 \cdot 350$ & $-0 \cdot 252$ & $0 \cdot 350$ & 30 & 90 & $29 \cdot 862$ & $90 \cdot 205$ & $-0 \cdot 138$ & $0 \cdot 205$ \\
\hline $50 \cdot 152$ & $9 \cdot 899$ & $0 \cdot 152$ & $-0 \cdot 101$ & 50 & 10 & 49.924 & $10 \cdot 026$ & -0.076 & 0.026 \\
\hline $50 \cdot 192$ & 29.986 & $0 \cdot 192$ & -0.014 & 50 & 30 & $50 \cdot 081$ & $30 \cdot 002$ & 0.081 & $0 \cdot 002$ \\
\hline $50 \cdot 374$ & $49 \cdot 855$ & 0.374 & $-0 \cdot 145$ & 50 & 50 & $50 \cdot 138$ & 49.986 & $0 \cdot 138$ & -0.014 \\
\hline $50 \cdot 043$ & $70 \cdot 247$ & 0.043 & $0 \cdot 247$ & 50 & 70 & $49 \cdot 867$ & $70 \cdot 351$ & $-0 \cdot 133$ & 0.351 \\
\hline $49 \cdot 310$ & $90 \cdot 828$ & $-0 \cdot 690$ & $0 \cdot 828$ & 50 & 90 & $49 \cdot 297$ & $90 \cdot 832$ & -0.703 & 0.832 \\
\hline $70 \cdot 465$ & $9 \cdot 691$ & 0.465 & $-0 \cdot 309$ & 70 & 10 & $70 \cdot 486$ & $9 \cdot 612$ & 0.486 & $-0 \cdot 388$ \\
\hline $70 \cdot 228$ & $29 \cdot 820$ & $0 \cdot 228$ & $-0 \cdot 180$ & 70 & 30 & $70 \cdot 144$ & 29.834 & 0.144 & $-0 \cdot 166$ \\
\hline $70 \cdot 805$ & $49 \cdot 414$ & $0 \cdot 805$ & -0.586 & 70 & 50 & $70 \cdot 581$ & $49 \cdot 561$ & 0.581 & -0.439 \\
\hline 69.888 & $70 \cdot 019$ & $-0 \cdot 112$ & 0.019 & 70 & 70 & 69.920 & 69.981 & -0.080 & -0.019 \\
\hline $69 \cdot 645$ & $90 \cdot 405$ & $-0 \cdot 355$ & 0.405 & 70 & 90 & 69.539 & 90.553 & $-0 \cdot 461$ & 0.553 \\
\hline 90.235 & $9 \cdot 811$ & 0.235 & $-0 \cdot 189$ & 90 & 10 & $90 \cdot 229$ & 9.768 & 0.229 & $-0 \cdot 232$ \\
\hline $90 \cdot 110$ & $29 \cdot 902$ & $0 \cdot 110$ & -0.098 & 90 & 30 & $90 \cdot 253$ & $29 \cdot 745$ & 0.253 & $-0 \cdot 255$ \\
\hline 89.908 & $50 \cdot 138$ & -0.092 & $0 \cdot 138$ & 90 & 50 & $90 \cdot 103$ & 49.966 & $0 \cdot 103$ & -0.034 \\
\hline 89.541 & $70 \cdot 267$ & -0.459 & $0 \cdot 267$ & 90 & 70 & 89.770 & $70 \cdot 120$ & $-0 \cdot 230$ & $0 \cdot 120$ \\
\hline 88.999 & $90 \cdot 625$ & $-1 \cdot 001$ & 0.625 & 90 & 90 & $89 \cdot 052$ & $90 \cdot 689$ & -0.948 & $0 \cdot 689$ \\
\hline
\end{tabular}

Table 3. Results for the analysis of the 'sample' solutions containing either theobromine or caffeine, but analyzed as their possible mixtures.

\begin{tabular}{|c|c|c|c|c|c|c|c|c|c|}
\hline \multicolumn{6}{|c|}{ Results from least squares } & \multicolumn{4}{|c|}{ Results from determinant ratio } \\
\hline c found & $t$ found & $\Delta c$ & $\Delta t$ & c nom & t nom & c found & $t$ found & $\Delta c$ & $\Delta t$ \\
\hline $0 \cdot 344$ & 9.790 & $0 \cdot 344$ & -0.210 & 0 & 10 & $0 \cdot 209$ & $9 \cdot 877$ & $0 \cdot 209$ & -0.123 \\
\hline$-0 \cdot 192$ & $30 \cdot 402$ & -0.192 & 0.402 & 0 & 30 & $0 \cdot 130$ & $30 \cdot 067$ & $0 \cdot 130$ & 0.067 \\
\hline 0.223 & $50 \cdot 043$ & 0.223 & 0.043 & 0 & 50 & $0 \cdot 400$ & $49 \cdot 808$ & $0 \cdot 400$ & -0.192 \\
\hline 0.669 & $69 \cdot 651$ & 0.669 & -0.349 & 0 & 70 & 0.541 & $69 \cdot 680$ & 0.541 & -0.320 \\
\hline 0.289 & $89 \cdot 912$ & 0.289 & -0.088 & 0 & 90 & 0.209 & 89.913 & $0 \cdot 209$ & -0.087 \\
\hline 9.872 & 0.063 & -0.128 & 0.063 & 10 & 0 & 9.936 & -0.007 & -0.064 & -0.007 \\
\hline $30 \cdot 198$ & -0.137 & $0 \cdot 198$ & -0.137 & 30 & 0 & $30 \cdot 138$ & $-0 \cdot 130$ & $0 \cdot 138$ & $-0 \cdot 130$ \\
\hline $50 \cdot 311$ & $-0 \cdot 355$ & $0 \cdot 311$ & -0.355 & 50 & 0 & $50 \cdot 252$ & $-0 \cdot 367$ & 0.252 & -0.367 \\
\hline $70 \cdot 198$ & $-0 \cdot 140$ & $0 \cdot 198$ & $-0 \cdot 140$ & 70 & 0 & $70 \cdot 082$ & $-0 \cdot 104$ & 0.082 & $-0 \cdot 104$ \\
\hline $90 \cdot 405$ & $-0 \cdot 282$ & 0.405 & -0.282 & 90 & 0 & $90 \cdot 278$ & $-0 \cdot 239$ & 0.278 & -0.239 \\
\hline
\end{tabular}

high quality of the spectrometer used, and the care we took to reduce several probable errors.

\section{Some disturbing observations}

Table 1 shows the results obtained for the baselines, when analysed for caffeine and theobromine. These measurements are here placed together for ease of analysis, but were actually fairly evenly distributed over the data set. Each baseline measurement follows flushing the cell with buffer, and resetting the baseline accordingly, so that no accumulation of instrumental drift is involved. We see that these baselines all analyze as containing less than about 
$0.7 \mu \mathrm{M}$ of either caffeine or theobromine, with an absolute deviation $|c|$ or $|t|$ of $<0.6 \mu \mathrm{M}$ for the determinant analysis, and $<0.7 \mu \mathrm{M}$ for least squares.

We note that all large values of $\Delta c$ and $\Delta t$ occur in pairs, where the errors in $c$ and $t$ are of similar magnitude but of opposite sign. For example, the last base-line analysis listed in table 1 shows $\Delta c=$ $+0.66 \mu \mathrm{M}$ and $\Delta t=-0.60 \mu \mathrm{M}$ for least squares analysis, and $\Delta c=+0.52 \mu \mathrm{M}$ and $\Delta t=-0.48 \mu \mathrm{M}$ for the determinant ratio method.

This suggests that these results do not reflect an actual presence of either caffeine or theobromine in the baseline solutions; it would be hard to rationalize such large negative concentrations anyway. Instead, these results appear to be artifacts of the data processing, which can interpret a small absorbance deviation in terms of the difference between two much larger absorbances of two different mixture components. In the jargon of matrix algebra, one might consider this a case of a spectrometrically ill-conditioned problem.

Say that we have a small baseline hump, either positive or negative, in the region between 250 and $290 \mathrm{~nm}$. Since the difference between the molar absorptivities of caffeine and theobromine is at most some $17 \%$, and is primarily localized in this area, a numerical analysis can readily misinterpret such a hump as the difference between two much larger

Table 4. The data used in table 3 but now analysed for either caffeine or theobromine rather than their mixture.

\begin{tabular}{|c|c|c|c|c|}
\hline \multicolumn{5}{|c|}{ Theobromine only } \\
\hline \multicolumn{3}{|c|}{$\begin{array}{l}\text { Results from } \\
\text { least squares }\end{array}$} & \multicolumn{2}{|c|}{$\begin{array}{l}\text { Results } \\
\text { from } 273 \mathrm{~nm}\end{array}$} \\
\hline t found & $\Delta t$ & $t$ nom & $t$ & $\Delta t$ \\
\hline $10 \cdot 089$ & $0 \cdot 089$ & 10 & $10 \cdot 054$ & $0 \cdot 054$ \\
\hline $30 \cdot 235$ & $0 \cdot 235$ & 30 & $30 \cdot 176$ & $0 \cdot 176$ \\
\hline $50 \cdot 237$ & 0.237 & 50 & $50 \cdot 145$ & $0 \cdot 145$ \\
\hline $70 \cdot 233$ & $0 \cdot 233$ & 70 & $70 \cdot 137$ & $0 \cdot 137$ \\
\hline $90 \cdot 163$ & $0 \cdot 163$ & 90 & $90 \cdot 090$ & $0 \cdot 090$ \\
\hline \multicolumn{5}{|c|}{ Caffeine only } \\
\hline \multicolumn{3}{|c|}{$\begin{array}{l}\text { Results from } \\
\text { least squares }\end{array}$} & \multicolumn{2}{|c|}{$\begin{array}{l}\text { Results } \\
\text { from } 273 \mathrm{~nm}\end{array}$} \\
\hline c found & $\Delta c$ & c nom & $c$ & $\Delta c$ \\
\hline $9 \cdot 945$ & -0.055 & 10 & 9.927 & -0.073 \\
\hline $30 \cdot 041$ & $0 \cdot 041$ & 30 & 29.981 & -0.019 \\
\hline 49.904 & $-0 \cdot 096$ & 50 & $49 \cdot 815$ & $-0 \cdot 185$ \\
\hline $70 \cdot 037$ & $0 \cdot 037$ & 70 & 69.955 & -0.045 \\
\hline $90 \cdot 082$ & $0 \cdot 082$ & 90 & 89.991 & -0.009 \\
\hline
\end{tabular}

concentrations of these two species, of alternate signs, especially when there is no corresponding baseline hump in the wavelength region below $240 \mathrm{~nm}$ where the two spectra are near-identical. This seems to be, indeed, what happens here.

For a baseline, a possible solution might be to limit the analysis to physically realizable, i.e. nonnegative concentrations, as can be done by replacing the linear least squares routine LinEst by a nonlinear one, such as Excel's Solver, to which constraints can be added. This, however, would only work for baselines, not for actual samples. It is likely that this inverse cancellation effect may be one of the ultimate limits to spectroscopic mixture analysis when some of the mixture components have quite similar spectra. (In computer jargon, cancellation occurs when two large numbers are so similar that their difference is distorted when computed with finite numberlength. Here we have the inverse process, where the numerical analysis of a small signal makes up a difference between much larger, non-existing quantities.) Unfortunately, the usual tricks to make a mathematically ill-conditioned least squares problem behave, such as singular value decomposition, or using extended numberlength, do not provide any relief in this case, because the errors do not originate in computer operations (such as matrix inversion) but in the experimental data. Moreover, the determinant ratio method appears to be just as vulnerable to this effect.

The data in table 2 list the results for mixtures. We see that the resulting absolute errors (with re-

Table 5. The data used in table 1 but now analysed for either caffeine or theobromine rather than their mixture.

\begin{tabular}{|c|c|c|c|c|}
\hline \multicolumn{5}{|c|}{ Single component analysis } \\
\hline \multicolumn{3}{|c|}{ Results from } & \multirow{2}{*}{\multicolumn{2}{|c|}{$\begin{array}{c}\text { Results } \\
\text { from } 273 \mathrm{~nm}\end{array}$}} \\
\hline least squ & & c nom, & & \\
\hline c found & $t$ found & t nom & c found & $t$ found \\
\hline $0 \cdot 007$ & $0 \cdot 006$ & 0 & $0 \cdot 000$ & $0 \cdot 000$ \\
\hline $0 \cdot 035$ & $0 \cdot 034$ & 0 & 0.065 & 0.055 \\
\hline $0 \cdot 000$ & $0 \cdot 000$ & 0 & $0 \cdot 000$ & $0 \cdot 000$ \\
\hline 0.007 & $0 \cdot 007$ & 0 & 0.023 & $0 \cdot 019$ \\
\hline$-0 \cdot 004$ & $-0 \cdot 004$ & 0 , & $0 \cdot 001$ & 0.001 \\
\hline$-0 \cdot 035$ & $-0 \cdot 030$ & 0 & $-0 \cdot 028$ & $-0 \cdot 023$ \\
\hline$-0 \cdot 045$ & $-0 \cdot 040$ & 0 & $-0 \cdot 043$ & -0.037 \\
\hline$-0 \cdot 056$ & $-0 \cdot 050$ & 0 & $-0 \cdot 065$ & -0.055 \\
\hline$-0 \cdot 004$ & $-0 \cdot 003$ & 0 & $-0 \cdot 004$ & $-0 \cdot 003$ \\
\hline$-0 \cdot 004$ & $-0 \cdot 003$ & 0 & $-0 \cdot 004$ & -0.003 \\
\hline $0 \cdot 026$ & $0 \cdot 023$ & 0 & $0 \cdot 018$ & $0 \cdot 015$ \\
\hline$-0 \cdot 019$ & $-0 \cdot 020$ & 0 & $-0 \cdot 060$ & $-0 \cdot 050$ \\
\hline
\end{tabular}


spect to the nominal concentrations) for the least squares method are up to $1.0 \mu \mathrm{M}$ in caffeine, i.e. more than $1 \%$ of the nominal amount present, and are similar $(0.95 \mu \mathrm{M})$ for the determinant ratio. Again, these rather large errors all occur in pairs, where the signs of the errors $\Delta c$ and $\Delta t$ are opposite, while their magnitudes are similar. We therefore believe them to be ghost concentrations resulting from inverse cancellation.

The samples in table 3 contain only caffeine or theobromine, but not both, and therefore provide a way to establish whether inverse cancellation is, indeed, a reasonable interpretation. In table 3 we have analysed these 10 solutions assuming that they contain both mixture components, but we can also analyse these same absorbance data in terms of just the one species they are known to contain. The results of such an analysis are shown in table 4 . While these are only a few solutions, the results appear to provide a striking confirmation of our assumption, because the analysis results in table 4 , where the possibility of inverse cancellation is cutoff, are much closer to the mark.

We see the same when we revisit the baseline solutions, as done in table 5 , but this time analyse them in terms of either caffeine or theobromine contents, i.e. only in terms of a single component. Again, the one-component analysis makes it impossible for the software to blow up a small deviation as a difference between two much larger, near-equal absorbances of two different species. Accordingly, we compute caffeine and theobromine concentrations that are about an order of magnitude smaller than those listed in table 1 , for these very same data sets. With one trivial exception, they have the same signs, as one would expect for spectrometrically similar compounds.

Finally, we revisit the mixture results. There are many possible sources of error in these data, but few can lead to the correlation we have observed so far in the baseline and single standard solutions. So we look for tell-tale signs in the mixtures, by comparing the sum $(\Delta c+\Delta t)$ with the individual values of $\Delta c$ and $\Delta t$. We find that the standard deviations in the sums $(\Delta c+\Delta t)$ are always smaller than those in $\Delta c$ and $\Delta t$, often by a factor of three to four.

\section{Synthesis}

When organic chemists use instrumental methods to identify an active ingredient, they often follow this up by a synthesis, in order to confirm that the presumed compound indeed has the asserted functionality. In the present case, we seek a similar confirmation by generating a baseline that has the purported noise components, and by then checking whether this indeed can lead to the observed, erroneous data analysis results.

When we plot the difference between the two theobromine and caffeine standard spectra, and analyse this curve as a mixture, we find, not surprisingly, $c=1.0 \mu \mathrm{M}$ and $t=-1.0 \mu \mathrm{M}$. However, when analysed individually for either $c$ or $t$, by least squares we obtain $c=0.15 \mu \mathrm{M}$ or $t=0.13 \mu \mathrm{M}$. The results using the determinant method, i.e., based on just two or one wavelengths, are quite similar: $c=1.0 \mu \mathrm{M}$ and $t=-1.0 \mu \mathrm{M}$ when analyzed as a mixture, and $c=0.19 \mu \mathrm{M}$ or $t=0.16 \mu \mathrm{M}$ when analysed individually.

Finally, when we replace the difference spectrum by a crude approximation, a single Gaussian peak $0.0017 \exp \left[-0.003(\lambda-273)^{2}\right]$ as shown in figure 3 , we again obtain quite similar results. It is clear that inverse cancellation, i.e. the making up of differ-

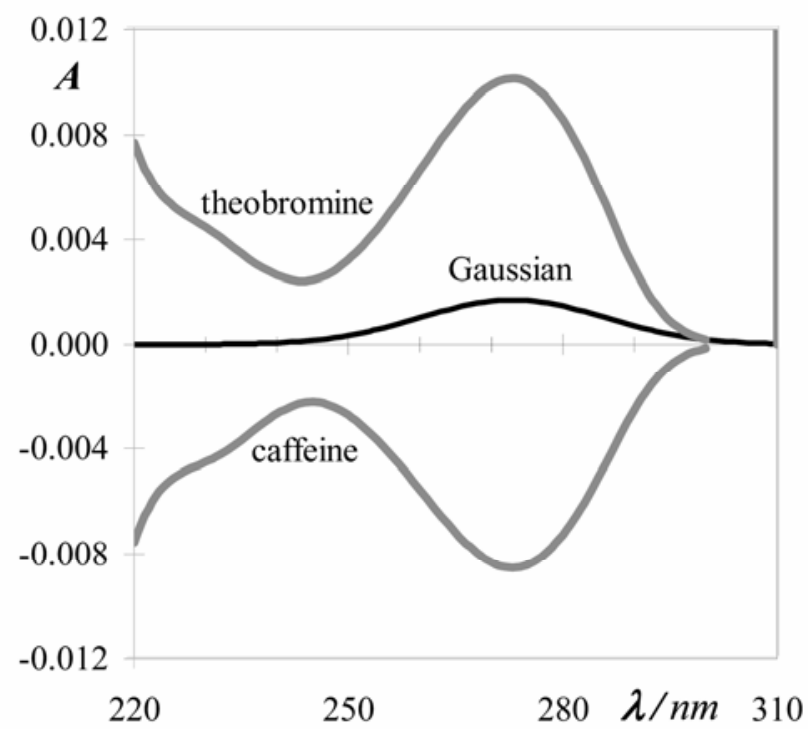

Figure 3. The Gaussian peak $0.0017 \exp [-0.003(\lambda-$ $73)^{2}$ ] (black curve labelled Gaussian, $\lambda=$ wavelength in $\mathrm{nm})$, when analysed as a possible mixture of caffeine and theobromine, yields $c=-1.001 \mu \mathrm{M}$ and $t=+1.008 \mu \mathrm{M}$ (gray curves) for both the least squares analysis, and the determinant ratio method. When analysed separately for either $c$ or $t$, the same analysis yields $c=+0.153 \mu \mathrm{M}$ or $t=+0.137 \mu \mathrm{M}$ with least squares, or $c=+0.1993 \mu \mathrm{M}$ or $t=+0.167 \mu \mathrm{M}$ for the wavelength ratio at $273 \mathrm{~nm}$. Clearly, the mixture analysis of an artificial mole hill conjures up two ghost mountains. 
ences between larger quantities not related to real, physical entities, can and does indeed occur.

\section{Discussion and conclusion}

This work was started as a somewhat routine effort to see how far one can push the simultaneous spectrometric determination of a mixture of species with quite similar molar absorptivities, and to compare the least squares and determinant methods when applied to data with fairly low levels of random errors. It has given us two answers. (i) In the present case, one can determine the concentrations of caffeine and theobromine in the concentration range between 0 and $100 \mu \mathrm{M}$ to a standard deviation of about $0 \cdot 34 \mu \mathrm{M}$ (and a corresponding 99\% confidence level of about $\pm 1 \mu \mathrm{M}$ ) with either method. (ii) More interestingly, these measurements contain an unexpected source of errors. On the other hand, the sum concentrations of caffeine plus theobromine can be determined with much lower standard deviations, of about 0.13 and $0.10 \mu \mathrm{M}$ for the least squares and determinant method respectively.

The reason that the determinant method yields somewhat better results is, apparently, that the two wavelengths chosen for the determinant analysis are those with the largest discrimination between the caffeine and theobromine absorptivities, whereas the least squares method mixes these with data where that distinction is much smaller. Moreover, as the noise level in our experimental data is very small, no doubt due to filtering inside the spectrophotometer, there is little advantage to using an overdetermined system, beyond providing uncertainty estimates of its parameters.

If the molar absorptivities of caffeine and theobromine were identical, it would be clear that one could not use spectrometry to determine their individual concentrations, but that only their sum would be accessible. When the molar absorptivities differ by a small amount, small fluctuations in the sample or baseline data can sometimes be misinterpreted in terms of non-existing concentration differences, while hardly affecting the sum concentrations. This appears to be happening here. We want to emphasize two points. First, while we have taken precautions to reduce experimental baseline drift, one can never guarantee its total absence. That is where the simulation comes in, because the simulated curves have zero baseline drift, and yet produce the same effect. Secondly, we have made sure that our results are not a matter of computational round-off either. When we repeated the least squares calculations with a numberlength of 200 decimals $^{2}$ (instead of the IEEE-754 standard 15 decimals of double precision used in Excel), we obtained identical results.

We therefore conclude that, for the spectrometric analysis of compounds with very similar molar absorptivities, we need to meet much more stringent requirements of data acquisition, so that the occurrence of misinterpretable fluctuations in both sample and baseline measurements is reduced. Furthermore, this problem is bound to become more severe when one tries to analyse more complex mixtures of spectrometrically similar compounds, such as of caffeine, theobromine, and their close relative theophylline, i.e. 1,3-dimethylxanthine.

While I am unaware of this having been pointed out, in this or other analytical contexts, I would be surprised if inverse cancellation has not been noticed before, though most likely under a quite different name. Its presence sets rather high experimental requirements, in terms of baseline reproducibility and instrumental drift, on a simultaneous analysis of spectrometrically similar compounds in their mixture. In the present example of a binary mixture, that requirement can be up to $1 / r$ times higher than that for a single-component analysis, where $r$ is the relative difference $2\left(a_{c}-a_{t}\right) /\left(a_{c}+a_{t}\right)$ of the molar absorbances at their wavelength of greatest difference, $a_{c}-a_{t}$. In other words: we have observed a gradual transition from what is possible with dissimilar spectra to what is impossible with identical ones, depending on their degree of similarity. As the Greek saying goes: $\pi \alpha v \tau \alpha \rho \varepsilon$, panta rei, everything flows, gradually.

\section{Acknowledgements}

The author thanks Suresh P. Jones and the Coles Fund of the Research Corporation for assistance with gathering preliminary data during a 2005 summer fellowship.

\section{References}

1. de Levie R 2008 Advanced Excel for Scientific Data Analysis (Oxford Univ. Press, New York) 2nd edn, section 3.7.

2. This routine, $x L S 0$, is freely downloadable from http ://www.bowdoin.edu/ rdelevie/excellaneous, and is based on the work of Leonardo Volpi, specifically his free Excel add-in software package Xnumbers.ddl, see his website at http://digilander.libero.it/ foxes/. 